\title{
Rise of the machines
}

\author{
Machine learning holds great potential to accelerate materials research. Many domains in \\ materials science are benefiting from its application, but several challenges persist, and it remains \\ to be seen whether the field will live up to the hype that surrounds it.
}

The age of machines is upon us. As we present our Focus Issue on machine learning in materials science, we are well aware that an algorithm could have written a reasonable opening Editorial for it. After all, it would not be its first pass at writing articles or, for that matter, even books.

You can ask Alexa or Siri, and it will use its machinelearning algorithm to find you some articles about the benefits and perils of artificial intelligence. Depending on your past searches and what they reveal about your interests, it might go on to speculate about whether there is too much hype around the ability of machine-learning tools to outperform humans.

However, there is no doubt that machine learning is impacting all domains of science, and materials science is no exception. In this Focus Issue, and in other articles in the journal, we explore how machine learning is pushing forward materials research, what progress we can realistically expect, and what researchers should pay attention to in order to ensure their machine-learning algorithms work the way they are designed to.

To set the scene, Rampi Ramprasad and colleagues survey in a Review the key components of machine learning for materials science, from protocols for data acquisition and management to strategies for autonomous experimentation. They also reflect on the most pressing open challenges, which include the need for standardized protocols, benchmarking datasets, and the open sharing of machine-learning code and data.

This machine-learning toolbox is already being fruitfully applied across virtually all topics in materials science. It is used for the design of photonic devices, as detailed in a Review by Jonathan Fan and colleagues. Its ability to navigate polymers' complex structurefunction landscapes can be exploited to engineer high-performance polymers, as Adam Gormley and Michael Webb describe in a Comment. When applied to alloys, machine learning pushes progress for the optimization of materials that range from metallic glasses to high-entropy alloys and structural materials, as explored in a Review by Gus Hart and colleagues.

Further, machine learning accelerates and improves the synthesis of nanoparticles with diverse properties, which is the topic of a Review by Eugenia Kumacheva and colleagues. As Olga Troyanskaya and co-authors discuss in their Review, machine learning has also become a key tool for the interrogation of complex and large biomedical datasets, enabling the study of multicellular complexity and the development of individualized therapies. Because it can be applied to vastly different problems, machine learning can catalyse crosspollination between disciplines, as Carla Gomes and collaborators describe in a Comment on the synergies between its application to sustainability and materials science problems.

One promising application of machine learning is in text mining, to extract and integrate information from articles and other documents into structured datasets. This feat is particularly important for data that are complex and difficult to organize, such as biomaterials data, as Osnat Hakimi and collaborators argue in a Comment. Another important trend is the push for autonomous systems that can perform experiments, measure the outcome and make decisions on the next iteration. This approach is especially advantageous for experiments that require tedious manual optimization: one example among many is the tuning of superconducting qubits, a field in which, as Natalia Ares discusses in a Comment, machine learning might be a key enabler of scalability to a relevant number of qubits.

Like in other domains in science, to move from proof-of-principle demonstrations to real applications, synergies with industrial partners will be crucial. Muratahan Aykol and colleagues argue in a Comment that this is the case, in particular, for the application of machine learning to battery optimization, in which data obtained from testing under realistic conditions is essential. To explore the point of view of an industrial researcher, we talked to Patrick Riley, until recently an engineer at Google Research, who says that an important trend as the field matures is a shift to thinking about machine learning not as a separate component of a system but as a well-integrated gear.

It is likely that not all the promises of machine learning will come to fruition. Machine learning is a powerful tool, but it will be a long time before machine-learning algorithms will be able to match the passion and creativity that researchers, as highlighted by the depth and breadth of this issue, contribute to materials science. That is, perhaps, a good thing. 